Published in final edited form as:

Fertil Steril. 2020 October ; 114(4): 828-836. doi:10.1016/j.fertnstert.2020.03.036.

\title{
CONTRIBUTIONS TO PREMATURITY OF MATERNAL HEALTH CONDITIONS, SUBFERTILITY, AND ASSISTED REPRODUCTIVE TECHNOLOGY (ART)
}

\author{
Judy E. Stern, PhD ${ }^{\mathrm{a}}$, Chia-ling Liu, ScD, MPH ${ }^{\mathrm{b}}$, Sunah S. Hwang, MD, MPH, $\mathrm{PhD}^{\mathrm{c}}$, Dmitry \\ Dukhovny, MD, MPH ${ }^{d}$, Hafsatou Diop, MD, MPH ${ }^{b}$, Howard Cabral, PhD, MPH \\ aDepartment of Obstetrics \& Gynecology, Dartmouth-Hitchcock, Lebanon, NH \\ ${ }^{b}$ Bureau of Family Health and Nutrition, Massachusetts Department of Public Health, Boston, MA \\ 'Department of Pediatrics, University of Colorado School of Medicine, Aurora, CO \\ dDepartment of Pediatrics, Oregon Health \& Science University, Portland, OR \\ eDepartment of Biostatistics, Boston University School of Public Health, Boston, MA
}

\section{Abstract}

Objective: To determine the maternal demographic, health, and fertility variables underlying prematurity.

Design: Retrospective

Setting: SART CORS data linked to Massachusetts birth certificates and hospital stays.

Patients: We included 166,963 privately insured, singleton, first births to women $\geq 18$ years of age between 2004-2013. Deliveries were: ART when linked to SART CORS; medically assisted reproduction (MAR) when fertility treatment was indicated on the birth certificate; unassisted subfertile (USF) when there were indications of subfertility but no treatment; and fertile if none of the above.

\section{Intervention: None}

Main Outcome Measures: Late preterm birth (LPTB: 34-36 weeks) and early preterm birth (EPTB: <34 weeks) were compared to term deliveries ( $>37$ weeks). Covariates significantly influencing prematurity in binary analysis were modeled using multinomial logistic regression. Backward elimination and mediation analysis were used to determine the influence of single parameters on outcomes of others.

Corresponding Author: Judy E. Stern, PhD, Department of Obstetrics \& Gynecology, Dartmouth-Hitchcock, Lebanon, NH 03756, phone: 603-252-0696, judy.e.stern@ dartmouth.edu.

Publisher's Disclaimer: This is a PDF file of an unedited manuscript that has been accepted for publication. As a service to our customers we are providing this early version of the manuscript. The manuscript will undergo copyediting, typesetting, and review of the resulting proof before it is published in its final form. Please note that during the production process errors may be discovered which could affect the content, and all legal disclaimers that apply to the journal pertain.

Abstract presented as a prize paper candidate at the $75^{\text {th }}$ annual meeting of the American Society for Reproductive Medicine in Philadelphia, PA. October 2019. 
Results: LPTB was increased in the USF (adjusted odds ratio [AOR] 1.32, 95\% confidence interval [CI] 1.06-1.65) and ART (1.42, 1.30-1.56) but not MAR (1.16, 0.98-1.37). ETPB was increased in all (USF 1.67, 1.21-2.31; MAR 1.67, 1.31-2.12; ART 1.40, 1.21-1.61). Strongest effectors of prematurity (AOR) were placental problems (LPTB 4.02; EPTB 10.28), pregnancy hypertension (LPTB 2.14; EPTB 2.88), and chronic hypertension (LPTB 1.85; EPTB 2.79). Mediation analysis demonstrated significant indirect effect of placental problems for ART and subfertility.

Conclusion: The greatest effectors of prematurity were placental problems and hypertensive disorders. ART and to a lesser extent subfertility were both associated with preterm birth directly and indirectly mediated via placenta problems.

\section{Capsule:}

Subfertility and ART increased both late and early preterm birth. Placental problems are on the pathway to late preterm birth and early preterm birth for ART and subfertile deliveries.

\section{Keywords}

ART; subfertility; prematurity; placenta; mediation analysis; IVF

\section{Introduction}

It is now well known that singleton deliveries to assisted reproductive technology (ART)treated women are at greater risk of adverse birth outcomes than the general population. In particular, there is an increased risk for prematurity and low birthweight (1-4). Pregnancies to ART-treated women are themselves at greater risk and include increased rates of gestational diabetes, pregnancy hypertensive disorders (pregnancy hypertension, preeclampsia and eclampsia), and placental abnormalities (4-7).

Pregnancies and deliveries to women dealing with infertility who are not treated with ART have also been shown to be at increased risk for these same factors. Declercq et al. (3) found that a subfertile population had increased risk of gestational diabetes and pregnancy hypertension, preterm birth, and low birthweight. Similarly, Luke et al. (4) found an increase in placental problems, pregnancy hypertension, gestational diabetes, pregnancy associated bleeding, preterm birth, and low birthweight among the subfertile women. Reasons for these increases are not known but could involve both ART treatment parameters (7) and underlying infertility (8).

Prior studies have looked at the risk of prematurity by evaluating cohorts of ART and subfertile as compared to fertile individuals to see whether prematurity increased. However, given the increase in pregnancy associated risk factors in ART and subfertile deliveries, it is possible that some of these factors contribute directly to shortened gestation. In this study, our goal was to look more directly at the risk for prematurity, to evaluate a number of maternal health and pregnancy factors that could potentially influence this endpoint, and to assess whether subfertility and/or ART influence or are influenced by these maternal health and pregnancy conditions. 


\section{Methods}

This was a retrospective cohort study that used Massachusetts ART data linked to state vital records and hospital discharges.

\section{Data Sources:}

We used data from 1) the Society for Assisted Reproductive Technology Clinic Outcome Reporting System (SART CORS) and 2) the Massachusetts-based Pregnancy to Early Life Longitudinal (PELL) data system. SART CORS is a national registry containing cycle-based ART data from the majority of ART clinics in the US. PELL is an ongoing population-based data system that compiles information from birth certificates, fetal death certificates and corresponding delivery hospital discharge records and ongoing hospital utilization records for mothers and infants over time. A Memorandum of Understanding among SART, the Massachusetts Department of Public Health (MDPH), and the project principal investigators was executed before initiation of the project. Approval for this study was obtained from MDPH and the Dartmouth-Hitchcock Health Institutional Review Board.

The SART CORS database is used to collect ART cycle specific demographics, infertility diagnoses, ART treatment, pregnancy, and outcome data under the auspices of SART. Data are collected from more than $90 \%$ of US ART clinics and from all Massachusetts clinics. Data are entered by the clinics and reported to the Centers for Disease Control and Prevention in compliance with the Fertility Clinic Success Rate and Certification Act of 1992 (Public Law 102-493). The data in the SART CORS are validated annually with random clinics having on-site visits for chart review based on an algorithm for clinic selection. During these visits, data reported by the clinic are compared with information recorded in patients' charts. In 2014, data fields selected for validation were found to have discrepancy rates of $\mathbf{5 6 \%}(9)$.

The PELL data system has linked over 98\% of Massachusetts births and fetal deaths to corresponding hospital utilization data (hospital admissions, observational stays, and emergency room visits) for individual women and their children since 1998. PELL is a relational data system composed of individual databases linked together by randomlygenerated unique IDs for mothers and infants. The MDPH and the Massachusetts Center for Health Information and Analysis are custodians of the PELL data which are housed at MDPH.

\section{Linkage of SART CORS to PELL:}

The Massachusetts Outcome Study of Assisted Reproductive Technology (MOSART) database was constructed by linking the SART CORS and PELL data systems for all Massachusetts resident women delivering in Massachusetts hospitals. The starting date represents the earliest SART CORS data available for research. A deterministic five phase linkage algorithm methodology was used to link data for deliveries between July 1, 2004 (January 1, 2004 cycle starts with first deliveries in July) and December 31, 2013. Linkage was based on mother's date of birth, her first name and last name; father/partner's last name, baby's date of birth, plurality, and infant sex (10). The linkage rate for 2004-2013 data was 
90.2\% overall and $94.5 \%$ for deliveries in which both mother's zip code and clinic were located in Massachusetts. Consistency of the demographic data in the SART CORS as compared to birth certificates has been previously reported by us and shown to be high (11). While only $43.1 \%$ of deliveries identified through linkage as ART deliveries were identified as having had ART treatment via the checkbox on the birth certificate, those that did have the checkbox checked accurately reported ART treatment $75 \%$ of the time. Although we have no external validation for it, we assume that other medically assisted treatment also had the same ratios and thus that MAR treatment when indicated is accurate, although not all MAR treatment would have been captured.

\section{Patients:}

The study sample included first deliveries to women from the MOSART database who were $\geq 18$ years of age and who had a singleton live birth. The deliveries were to women with private health insurance for whom we had complete data (supplemental Figure 1). We excluded gestational carriers, multiparous women, and women who had stillbirths.

Multiparous women were excluded for two reasons: one was that including them would require addition of covariates for results of the prior delivery such as prior prematurity; the other is that including them would have required adjusting for multiple deliveries to the same woman.

\section{Outcome Measures:}

Gestational age was obtained from birth certificates and modified, when needed, by reported dates of last menstrual period. Gestational ages outside of the range of 17-44 weeks were considered as missing. Outcome groups included term deliveries ( 237 weeks gestation), late preterm deliveries (34-36 weeks gestation), and early preterm deliveries ( $<34$ weeks gestation).

\section{Fertility Groups:}

Women were classified as ART-treated if the delivery was linked to an ART cycle in the SART CORS database. The definition of the MOSART subfertile group has been previously described (12). In this study we divided this group into two groups. Women were classified as having medically assisted reproduction (MAR) if the birth certificate for the delivery indicated receipt of fertility treatment but the delivery was not linked to an ART cycle in SART CORS. They were classified as "unassisted subfertile" if they had a diagnosis of infertility (ICD9 628.9) in a hospital discharge, observational visit, or emergency room visit prior to the index delivery or if they had an unsuccessful ART cycle prior to the index delivery. Finally, women were classified as fertile if they did not fall into the ART-treated, MAR or unassisted subfertile groups.

\section{Covariates:}

We evaluated a large number of maternal conditions with potential to influence prematurity. Only those demonstrating significant differences $($ at $\mathrm{P}<0.05)$ were included in the final model. Covariates for analysis were obtained from birth certificates, hospital discharges, or a combination of the two. The following covariates were obtained from birth certificates: 
maternal and paternal demographics including age, race/ethnicity and education, country of origin, prior gravidity, year of delivery and infant sex. From hospital discharges we obtained maternal disease conditions of: uterine surgery prior to conception (CPT codes 57460, 57461, 57800, 58140, 58145, 58558, 58560, 58561, 59136, 59140, ICD codes 752.2, 761.4, $68.22,68.29,69.49)$, and conditions 5 years prior to conception including ectopic pregnancy/miscarriage $(633,634)$, leiomyoma (218), bleeding diseases of reproductive tract $(626,256)$, thyroid disease (240-246), electrolyte imbalance (276), psychological disorders (E50, V62, 296, 300, 301, 309, 311), overweight or obese status (278), any hospitalization, endometriosis (617), asthma (493), and drug abuse/addiction (292, 303-305). Five years was chosen for these conditions to provide a consistent timeframe for all years of study given that PELL data are available from 1998 and SART CORS data from 2004. A combination of birth certificates and hospital discharge records were used to define: chronic hypertension and diabetes, gestational diabetes, pregnancy hypertension/preeclampsia/eclampsia, pregnancy associated bleeding, and placental problems (abrupto placenta, placenta previa, vasa previa, and placenta accreta), other delivery complications including cephalopevic disproportion, breech/malpresentation, prolonged labor, dysfunctional labor, febrile, fetal distress, cord prolapse, rupture membrane premature, rupture member prolonged, and caesarian hysterectomy, and method of delivery.

\section{Statistical Analyses:}

Bivariate and multivariate multinomial logistic regressions were used to estimate the crude and adjusted associations between outcome (full-term, late preterm birth [LPTB], early preterm birth [EPTB]) and variables of interest (fertility status and covariates). Both crude and adjusted odds ratios (AOR) and 95\% confidence intervals (95\% CI) for each outcome comparing the LPTB and EPTB deliveries were reported. A full multivariable model included all 24 covariates shown in Tables 1 and 2. Backward eliminations were used to explore how presence and absence of a particular variable (i.e., fertility group and covariates) influenced the associations between preterm births and other variables. Using causalmed procedures in the statistical software SAS 9.4 (SAS, version 9.4. 2016. SAS Institute. Cary, NC.), mediation analyses were performed to quantify direct and indirect effects of fertility status on LPTBs and EPTBs. All other analyses were performed in SAS software 9.3 (SAS Institute, Cary NC).

\section{Results}

The study sample included 166,963 deliveries of which 155,997 were term, 8,210 were LPTB and 2,756 were EPTB. The fertility groups included 8,795 ART, 2,736 MAR, 1,317 unassisted subfertile, and 154,115 fertile deliveries. Table 1 shows the demographic characteristics that were significant in the crude analysis while Table 2 shows the significant maternal health, pregnancy and infant sex characteristics that were significant. Others that were not significant or related to delivery and not considered directly relevant to the fertility group pathway to prematurity were omitted. Furthermore, year of delivery was not a significant predictor and including it resulted in only minor changes to AORs; it was thus omitted from the final models. 
Adjusted odds ratios are shown in Tables 1 and 2. The covariates with greatest AORs were placental problems (4.02 LPTB and 10.28 EPTB) followed by pregnancy hypertensive disorders (2.14 LTPB, 2.88 EPTB), chronic hypertension (1.85 LPTB, 2.79 EPTB), and bleeding problems during pregnancy (1.49 LPTB, 2.50 EPTB). Compared to the fertile group, all other fertility groups had increased risk for prematurity: AORs for ART (1.42 LPTB, 1.40 EPTB), MAR (1.16 LPTB and 1.67 EPTB), and unassisted subfertility (1.32 LPTB, 1.67 EPTB).

We then asked the question of whether any of the covariates influence the effect of other covariates on prematurity. To evaluate this, we used backwards elimination of individual covariates from the model. Eliminating fertility groups did not affect any value. As examples, chronic diabetes with AOR of 1.72 and 2.02 for LPTB and EPTB, respectively had AOR of 1.73 and 2.04 for LPTB and EPTB, respectively following removal of fertility groups: chronic hypertension with AORs of 1.85 for LPTB and 2.79 for EPTB with all covariates had AORs of 1.85 and 2.79 for LPTB and EPTB, respectively with fertility group removed. By contrast, removing placental problems resulted in apparent changes in AORs for placental bleeding (1.49 for LPTB, and 2.50 EPTB with all covariates and 1.66 for LPTB and 3.27 for EPTB with placental problems removed) and the three fertility groups (AOR ART 1.42 for LPTB and 1.40 EPTB with all covariates and 1.53 and 1.66 for LPTB and EPTB, respectively with placental problems removed; AOR unassisted subfertile 1.32 for LPTB and 1.67 EPTB with all covariates and 1.35 and 1.79 for LPTB and EPTB, respectively with placental problems removed; AOR MAR 1.16 for LPTB and 1.67 for EPTB with all covariates and 1.17 and 1.71 for LPTB and EPTB, respectively with placental problems removed). Removing pregnancy hypertensive disorders did not change the risk for prematurity in the fertility groups.

To further explore whether placental problems influenced the effect of subfertility and ART on prematurity, we performed a mediation analysis. This analysis distinguishes between the direct effect of an exposure on an outcome and the indirect effect mediated through a third variable (Figure 1). In this case we were seeking to determine whether the influence of fertility group on prematurity, was mediated through placental problems. Table 3 shows the results of this analysis. The table presents total, direct and indirect effects. Although there is direct effect in all cases, the indirect effect was also significant for ART, MAR and unassisted subfertile groups. Calculation of percent mediation, which corresponds to the percentage of the total effect mediated through placental problems, demonstrated that for LPTB and EPTB ART deliveries, $15 \%$ and 32\% respectively were mediated through placental problems. A smaller proportion of MAR (12\%) and unassisted subfertile (14\%) groups' effects on EPTB were accounted for by an indirect effect however, the indirect effect on LPTB in the unassisted subfertile and MAR groups was not significant.

\section{Discussion}

The results of this study demonstrate that while ART and subfertility significantly increase the risk of prematurity, their effect is less than that of other factors such as placental problems and chronic and pregnancy associated hypertensive disorders. The results further 
demonstrate that a portion of the effect of ART and, to a lesser extent, subfertility is mediated through placental problems.

As Schieve first reported in 2004 (1), ART singletons have an increased risk of preterm delivery when compared with the general population. Dunietz et al. (2) further demonstrated that the risk of prematurity differs according to the infertility diagnosis with AORs of 1.24 for male infertility, 1.60 for female infertility, 1.49 for combined male and female infertility, and 1.26 for unexplained infertility. Deliveries to women with subfertility who have not been treated with ART have also been reported to have an increased risk of prematurity. DeClercq et al. (3) demonstrated an increased risk of prematurity in singleton deliveries of 1.53 for ART and 1.24 for subfertile women without ART treatment. The increases in prematurity in singleton deliveries to ART treated and subfertile women, shown in this study are consistent with these previous results.

Previous studies have also demonstrated an increase in a variety of pregnancy related abnormalities in both ART-treated and subfertile women carrying singleton pregnancies. Placental problems including placenta previa (5) and vasa previa (6) have been shown to be increased in ART pregnancies. A growing body of information also suggests that there may be changes in DNA methylation patterns in the placentas and cord blood following ART treatment (13-15). Luke et al. (4) demonstrated that placental problems are increased in ART-treated (AOR 2.81) and subfertile (AOR 1.44) pregnancies when compared with pregnancies in fertile women. These ART-treated and subfertile women when compared with fertile women also had increased pregnancy hypertension (AOR 1.22 ART; AOR 1.12 subfertile), gestational diabetes (AOR 1.41 ART; AOR 1.60 subfertile) and pregnancy associated bleeding (AOR 3.80 ART; AOR 1.67 subfertile). Infertility diagnosis as well as treatment may influence these risks. Stern et al. [Stern 2015] found gestational diabetes to be increased for women with ovulation disorders both with (AOR 2.17) and without (1.94) ART treatment. To our knowledge, the reasons for these increased risks have never been clearly determined, nor has it been determined what ART treatments or aspects of subfertility most contribute to these problems.

Our mediation analysis clearly demonstrated that, while a majority of the effect of ART on prematurity is through a direct effect, a considerable portion of the effect of ART on prematurity is mediated through placental problems. This is consistent with knowledge that placental abnormality can lead to prematurity (16-17). This is also consistent with the known increase in placental problems with ART. Luke et al (7) and suggest that certain ART parameters increase the risk for both placental problems and prematurity. Both fresh and frozen donor egg cycles increased prematurity compared with fresh autologous cycles. Fresh and frozen donor egg groups also manifested greater risk of gestational diabetes, pregnancy hypertension and placental problem. Determining which ART characteristics are most strongly associated with prematurity through mediation by placental problems will be the subject of future evaluation..

The unassisted subfertile group manifested greater odds of prematurity than did the MAR group. Given that this group is identified by hospitalizations and prior unsuccessful ART, this is not surprising. Most infertility is diagnosed and treated as outpatient problems. Only a 
small proportion of infertility treatment involves hospitalization and this occurs largely when the condition is of a more serious nature. The data from the unassisted subfertile group strongly suggest that more severe infertility is more strongly associated with prematurity than non-ART treatment. We assume that ART patients, by virtue of the fact that they have reached the most advanced treatment option for infertility also have more severe infertility than women in the MAR group. Nevertheless, the fact that considerably more of the prematurity is mediated through placental problems in the ART group as compared with the unassisted subfertile group, suggests that additional factors related to the ART treatment itself are instrumental in increasing the risk for prematurity through abnormal placentation.

This study has strengths and limitations. The strengths lie in the fact that MOSART is a large population-based database with maternal and infant hospital discharge records linked to birth certificate data, allowing for dyadic longitudinal analysis of maternal and infant health outcomes. The limitations include that this is a retrospective study and some important covariates such as smoking, diet, and BMI are either not available, are not available for all years, or have significant inaccuracies. Further, the fertile group may contain some unidentified subfertile deliveries, although these would serve only to decrease the magnitude of the observed differences. Covariates were identified by hospitalization for each condition, thus we were unable to identify women with any conditions diagnosed and treated solely on an outpatient basis. Because the unassisted subfertile group was defined by prior hospitalization with a recorded diagnosis of infertility and prior unsuccessful ART, this group may have more severe subfertility than a random population of infertile women. Finally, the study was performed in a single state, Massachusetts, and results may not be generalizable to all states and countries.

In summary, the data show that among our cohort, the greatest effectors of prematurity were placental problems and chronic and gestational hypertensive disorders. Subfertility and ART both increased LPTB and EPTB but to a lesser extent than these two conditions. In addition, we saw no indication that subfertility/ART directly altered the effect of other parameters. By contrast, removing placental problems appeared to affect the influence of subfertility and ART. Mediation analysis demonstrated that placental problems are on the pathway to LPTB and EPTB for a portion of ART and to a lesser extent, subfertile deliveries. The ART and subfertility parameters responsible for the effect on placental problems remains to be determined.

\section{Supplementary Material}

Refer to Web version on PubMed Central for supplementary material.

\section{Acknowledgements:}

SART wishes to thank all of its members for providing clinical information to the SART CORS database for use by patients and researchers. Without the efforts of our members, this research would not have been possible. Funding was obtained from NIH: RO1HD67270. 


\section{References}

1. Schieve LA, Ferre C, Peterson HB, Macaluso M, Reynolds MA, Wright VC. Perinatal Outcome Among Singleton Infants Conceived Through Assisted Reproductive Technology in the United States. Obstetrics \& Gynecology 2004; 103:1144-53 [PubMed: 15172846]

2. Dunetz GL, Holzman C, McKane P, Li C, Boulet SL, Todem D, et al. Assisted reproductive technology and the risk of preterm birth among primiparas. Fertil Steril 2015; 103:974-9. [PubMed: 25707336]

3. Declercq E, Luke B, Belanoff C, Cabral H, Diop H, Gopal D, et al. Perinatal Outcomes Associated with Assisted Reproductive Technology: the Massachusetts Outcomes Study of Assisted Reproductive Technologies (MOSART). Fertil Steril 2015; 103:888-95. [PubMed: 25660721]

4. Luke B, Gopal D, Cabral H, Stern JE, Diop H. Pregnancy, Birth, and Infant Outcomes by Maternal Fertility Status: The Massachusetts Outcomes Study of Assisted Reproductive Technology. American Journal Obstetrics and Gynecology 2017; 217:327.e1-14.

5. Romunstad LB, Romunstad PR, Sunde A, von Düring V, Skjærven R, Vatten LJ. Increased risk of placenta previa in pregnancies following IVF/ICSI; a comparison of ART and non-ART pregnancies in the same mother. Human Repro 2006: 21:2353-8.

6. Schachter M, Tovbin Y, Arieli S, Friedler S, Ron-El R, Sherman D. In vitro fertilization is a risk factor for vasa previa. Fertil Steril 2002; 78:642-3. [PubMed: 12215350]

7. Luke B, Brown MB, Wantman E, Seifer DB, Sparks AT, Lin PC, et al. Risk of prematurity and infant morbidity and mortality by maternal fertility status and plurality. J Assist Repro Genet 2019; 36:121-38 10.1007/s10815-018-1333-z

8. Stern JE, Luke B, Tobias M, Gopal D, Hornstein MD, Diop H. Adverse pregnancy and birth outcomes by infertility diagnoses with and without ART treatment. Fertil Steril 2015; 103:1438-45. [PubMed: 25813277]

9. Centers for Disease Control and Prevention. Assisted Reproductive Technology Clinic Success Rate Report 2014: Appendix A Technical Notes. ftp://ftp.cdc.gov/pub/Publications/art/ART-2014-ClinicReport-Full.pdf\#page=527

10. Kotelchuck M, Hoang L, Stern JE, Diop H, Belanoff C, Declercq ER. The MOSART database: Linking the SART CORS clinical database to the population-based Massachusetts PELL reproductive public health data system. Maternal and Child Health Journal 2014; 18:2167-78. [PubMed: 24623195]

11. Stern JE, Gopal D, Anderka M, Liberman R, Kotelchuck M, Luke B. Validation of birth outcomes in the SART CORS: Population-based analysis from the Massachusetts Outcome Study of Assisted Reproductive Technology (MOSART). Fertil Steril 2016; 106:717-22.e2. [PubMed: 27208695]

12. Declercq ER, Belanoff C, Diop H, Gopal D, Hornstein MD, Kotelchuck M, et al. Identifying women with indicators of subfertility in a statewide population database: operationalizing the missing link in assisted reproductive technology research. Fertil Steril 2014; 101:463-71. [PubMed: 24289994]

13. Nelissen EC, Dumoulin JC, Daunay A, Evers JL, Tost J, van Montfoort AP. Placentas from pregnancies conceived by IVF/ICSI have a reduced DNA methylation level at the H19 and MEST differentially methylated regions. Hum Reprod 2013; 28:1117-26. doi: 10.1093/humrep/des459 [PubMed: 23343754]

14. Litzky JF, Deyssenroth MA, Everson TM, Armstrong DA, Lamertini L, Chen J, et al. Placental imprinting variation associated with assisted reproductive technologies and subfertility. Epigenetics 2017; 12:653-61 doi: 10.1080/15592294.2017.1336589 [PubMed: 28621618]

15. Castillo-Fernandez JE, Loke YJ, Bass-Stringer S, Gao F, Xia Y, Wu H, et al. DNA methylation changes at infertility genes in newborn twins conceived by in vitro fertilisation. Genome Medicine 2017; 9:28 DOI 10.1186/s13073-017-0413-5 [PubMed: 28340599]

16. Fay-Petersen OM. The placenta in preterm birth. J Clin Pathol 2008; 61:1261-75. doi:10.1136/ jcp.2008.055244. [PubMed: 19074631]

17. Morgan TK. Role of the Placenta in Preterm Birth: A Review Am J Perinatol 2016; 33:258-66. doi: 10.1055/s-0035-1570379. [PubMed: 26731184] 


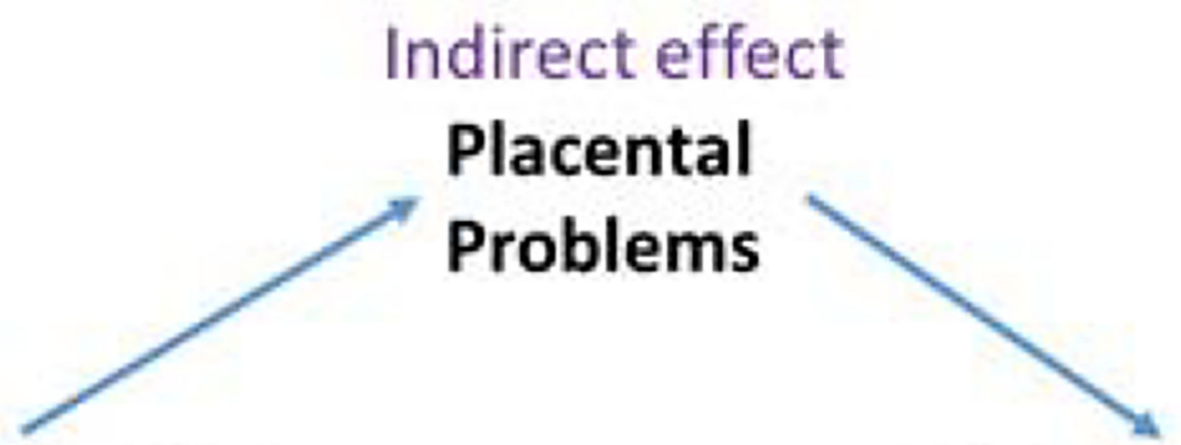

ART or Subfertility

Figure 1:

Mediation analysis. The mediation analysis shows results for mediation using placental problems as the mediator. 


\begin{tabular}{|c|c|c|c|c|c|c|c|c|c|c|c|c|c|c|c|c|c|c|c|c|}
\hline 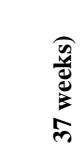 & 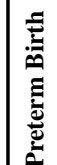 & $\begin{array}{l}z \\
\therefore 8 \\
2 \\
\alpha\end{array}$ & & 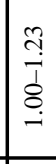 & 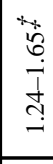 & 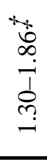 & & 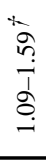 & & 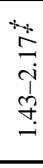 & 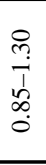 & 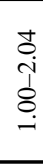 & $\begin{array}{l}\text { th } \\
\stackrel{5}{I} \\
= \\
=\end{array}$ & $\frac{\sqrt{n}}{\frac{n}{1}}$ & & & 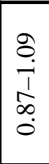 & $\begin{array}{l}0 \\
= \\
\overline{1} \\
\infty \\
\stackrel{0}{0}\end{array}$ & $\begin{array}{l}\text { I } \\
\bar{j} \\
+ \\
0 \\
0\end{array}$ & 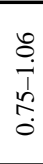 \\
\hline $\begin{array}{r}\ddot{\ddot{\Xi}} \\
-\ddot{\sigma}\end{array}$ & 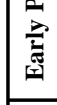 & $\frac{\pi}{0}$ & $\stackrel{\Xi}{ \pm}$ & $\exists$ & $\stackrel{\text { f }}{-}$ & 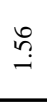 & $\stackrel{n}{g}$ & 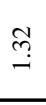 & ¿ & $\stackrel{?}{\stackrel{2}{-}}$ & 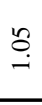 & $\stackrel{?}{\stackrel{f}{f}}$ & 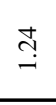 & $\bar{I}$ & $\stackrel{\square}{\square}$ & $\stackrel{\Psi}{\mathscr{D}}$ & à & ŏ & gे & \&̊ \\
\hline 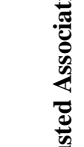 & 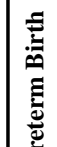 & 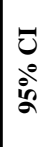 & & $\begin{array}{l}\infty \\
\stackrel{1}{0} \\
\stackrel{\vec{b}}{0} \\
\stackrel{0}{0}\end{array}$ & 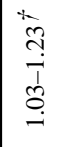 & 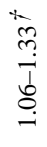 & $\begin{array}{l}\frac{\partial}{7} \\
\frac{1}{\infty} \\
0 \\
0 \\
0\end{array}$ & 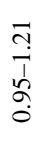 & & 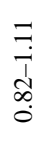 & 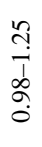 & $\begin{array}{l}\vec{T} \\
\stackrel{1}{n} \\
\hat{0} \\
0\end{array}$ & $\begin{array}{l}\exists \\
\frac{1}{1} \\
\frac{1}{0}\end{array}$ & 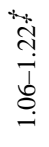 & & & 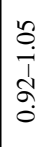 & 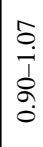 & 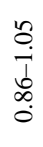 & $\begin{array}{l}\stackrel{\partial}{\circ} \\
\dot{1} \\
\text { के } \\
0\end{array}$ \\
\hline 字 & 总 & $\frac{\pi}{0}$ & $\stackrel{\Psi}{ \pm}$ & $\underset{\sim}{\sigma}$ & $\stackrel{9}{=}$ & $\stackrel{9}{\exists}$ & $\stackrel{\vec{\sigma}}{.}$ & 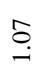 & ¿্口 & $\stackrel{\text { on }}{o}$ & $\exists$ & $\stackrel{\leftrightarrow}{o}$ & $\stackrel{t}{\stackrel{\Delta}{*}}$ & $\stackrel{?}{=}$ & 巳 & $\stackrel{\varpi}{\mathscr{D}}$ & $\begin{array}{l}\infty \\
\stackrel{0}{0}\end{array}$ & $\stackrel{\infty}{\circ}$ & ֶ̆ & $\stackrel{\circ}{\circ}$ \\
\hline 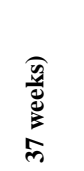 & 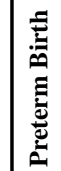 & $\begin{array}{l}\text { U } \\
8^{\circ} \\
\text { a }\end{array}$ & & 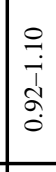 & |ct & 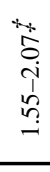 & 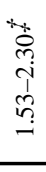 & 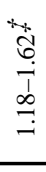 & & 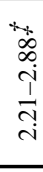 & $\begin{array}{l}\text { *o } \\
\& \\
0 \\
1 \\
\stackrel{0}{0} \\
0\end{array}$ & 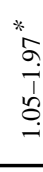 & $\frac{\text { th }}{n}$ & 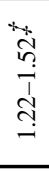 & & & 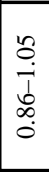 & \begin{tabular}{l}
$\stackrel{1}{1}$ \\
\multirow{1}{1}{} \\
$\hat{\sigma}$ \\
0
\end{tabular} & & 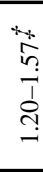 \\
\hline$\underset{\Xi}{\ddot{\Xi}}$ & 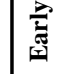 & 。ี & 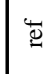 & $\underset{-}{\sigma}$ & $\stackrel{\text { qq }}{-}$ & $\stackrel{2}{i}$ & $\stackrel{\infty}{\rightarrow}$ & 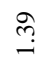 & 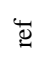 & त̂ & $\begin{array}{c} \pm \\
\stackrel{\infty}{0}\end{array}$ & $\stackrel{\text { J }}{\text { J }}$ & $\stackrel{?}{-}$ & 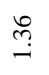 & 巳 & $\Phi$ & ŏ & 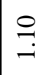 & $\stackrel{\oplus}{\rightarrow}$ & $\stackrel{\widehat{m}}{\rightarrow}$ \\
\hline 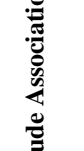 & 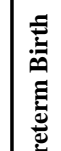 & $\begin{array}{l}\text { च } \\
0^{\circ} \\
\text { a }\end{array}$ & & 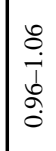 & 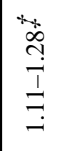 & 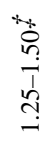 & 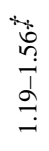 & & & 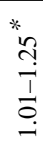 & $\begin{array}{l}* \\
2 \\
\infty \\
0 \\
1 \\
\infty \\
0 \\
0\end{array}$ & $\frac{\stackrel{7}{7}}{\stackrel{1}{t}}$ & $\begin{array}{l}\frac{1}{9} \\
\frac{1}{0} \\
\end{array}$ & 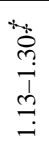 & & & $\begin{array}{l}\tilde{o} \\
\stackrel{\vec{T}}{\grave{d}} \\
\hat{\sigma}\end{array}$ & $\begin{array}{l}\stackrel{9}{1} \\
\overline{1} \\
\stackrel{0}{0}\end{array}$ & & 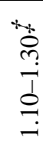 \\
\hline & 离 & 。 & $\stackrel{\varpi}{\varpi}$ & $\underset{-1}{8}$ & ঙ્̣ & 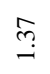 & $\stackrel{\sqrt[m]{7}}{2}$ & $\stackrel{\Im}{\exists}$ & $\stackrel{\square}{2}$ & $\cong$ & $\bar{a}$ & ஃ̊ & $\stackrel{?}{=}$ & $\overline{\mathrm{I}}$ & 崩 & $\stackrel{\Psi}{\mathscr{Z}}$ & so & $\stackrel{\bullet}{\leftrightarrow}$ & $\stackrel{9}{\leftrightarrows}$ & త్రి \\
\hline 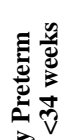 & $\Delta^{\circ}$ & ב & $\stackrel{n}{n}$ & $\because$ & $\vec{i}$ & 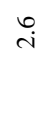 & $\hat{i}$ & $\overrightarrow{\mathrm{i}}$ & $\because$ & $\stackrel{\infty}{\dot{m}}$ & $\stackrel{?}{=}$ & กี & $\vec{i}$ & $\stackrel{\circ}{i}$ & $\stackrel{n}{\longrightarrow}$ & $\stackrel{n}{-}$ & $\stackrel{?}{?}$ & 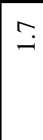 & $\stackrel{\circ}{i}$ & $\vec{i}$ \\
\hline
\end{tabular}

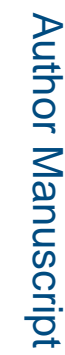

$\ddot{\sigma}$
$\frac{0}{0}$
$\frac{0}{\pi}$

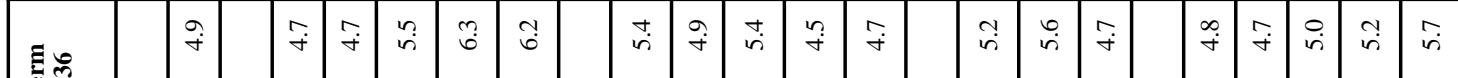

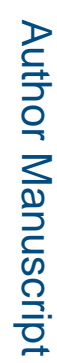

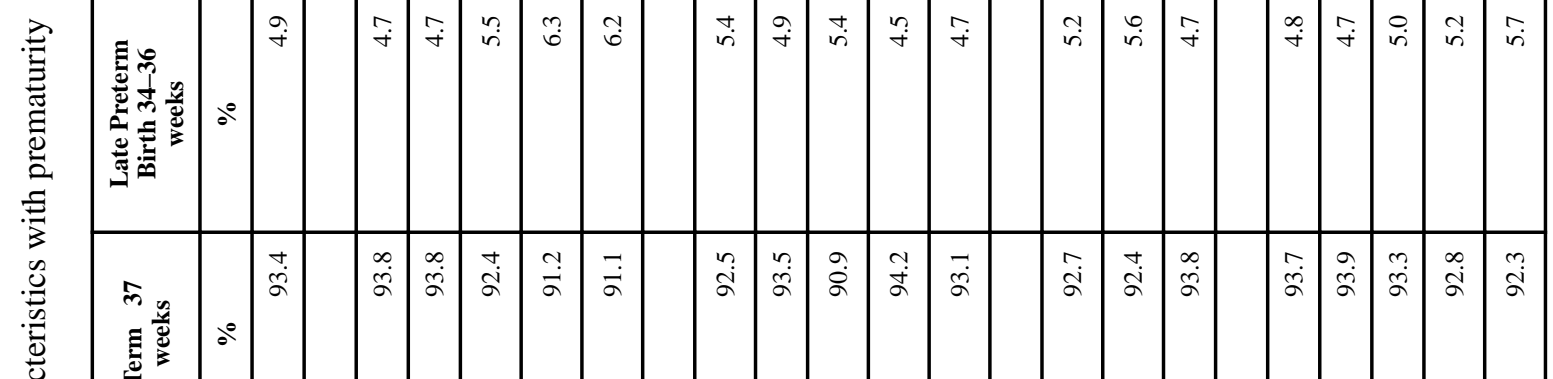

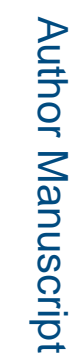

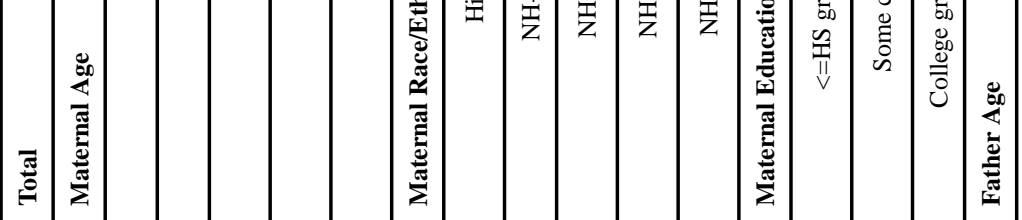

Fertil Steril. Author manuscript; available in PMC 2021 October 01. 


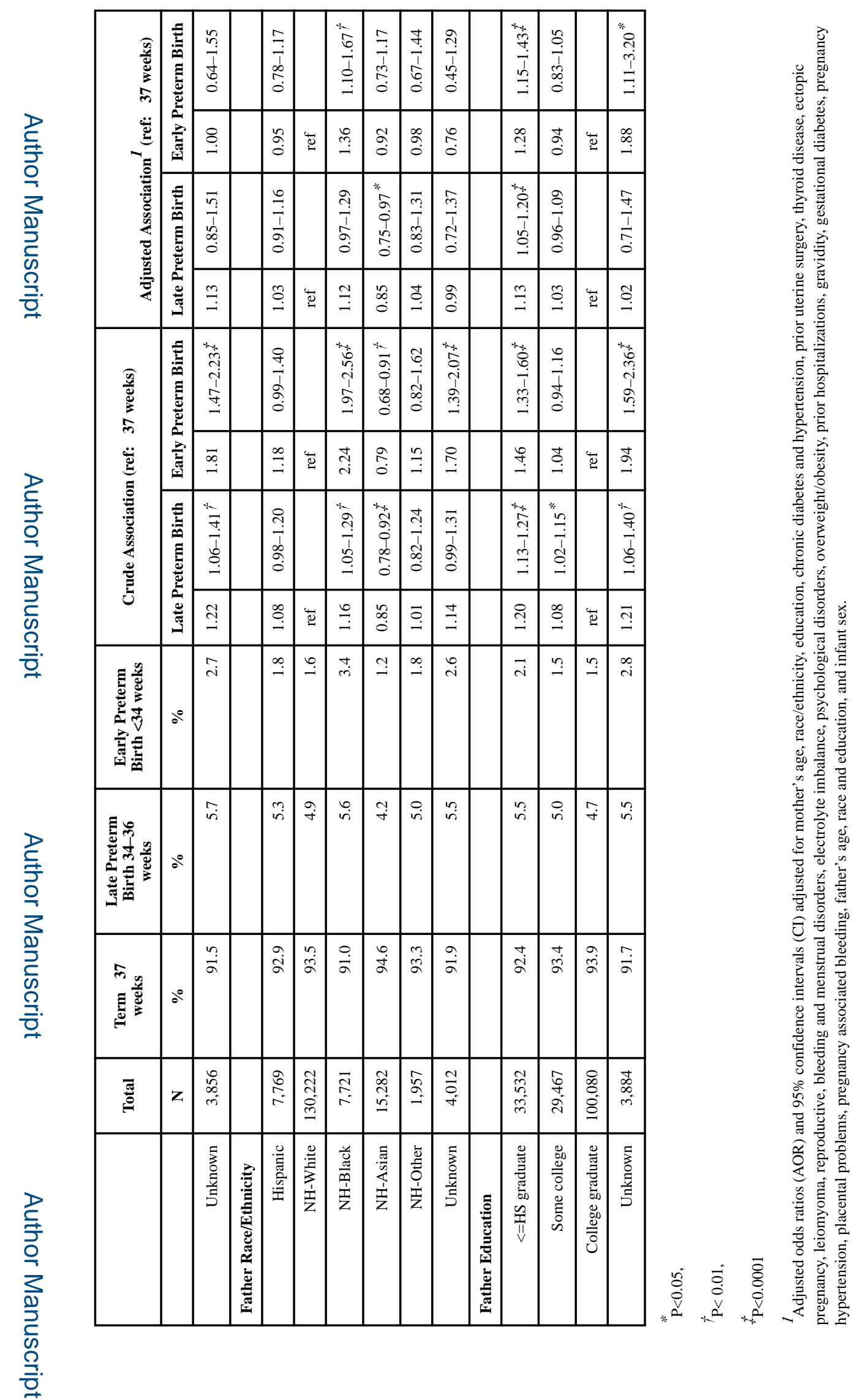

Fertil Steril. Author manuscript; available in PMC 2021 October 01. 


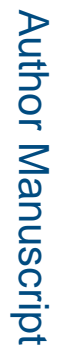

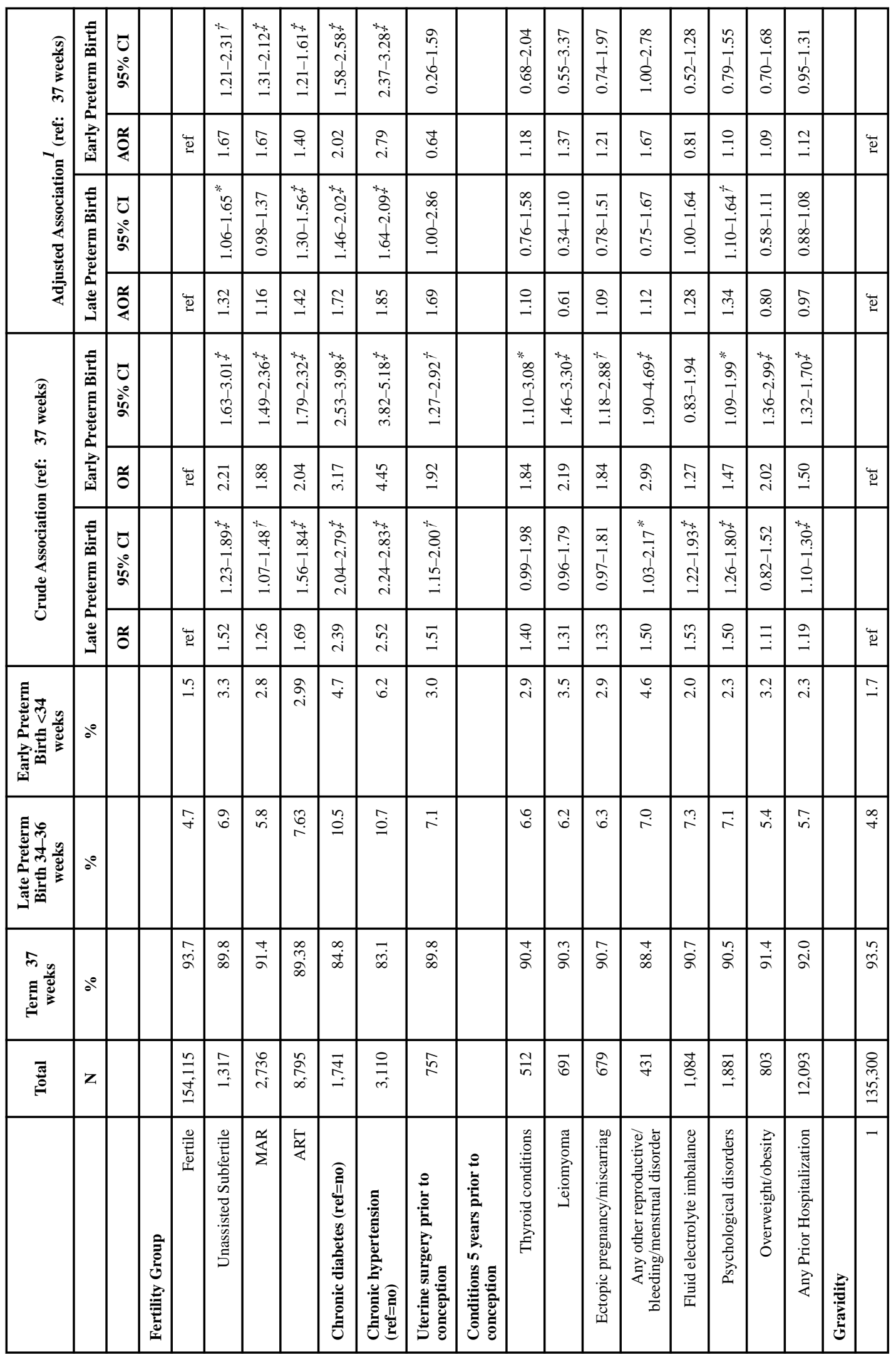

Fertil Steril. Author manuscript; available in PMC 2021 October 01. 


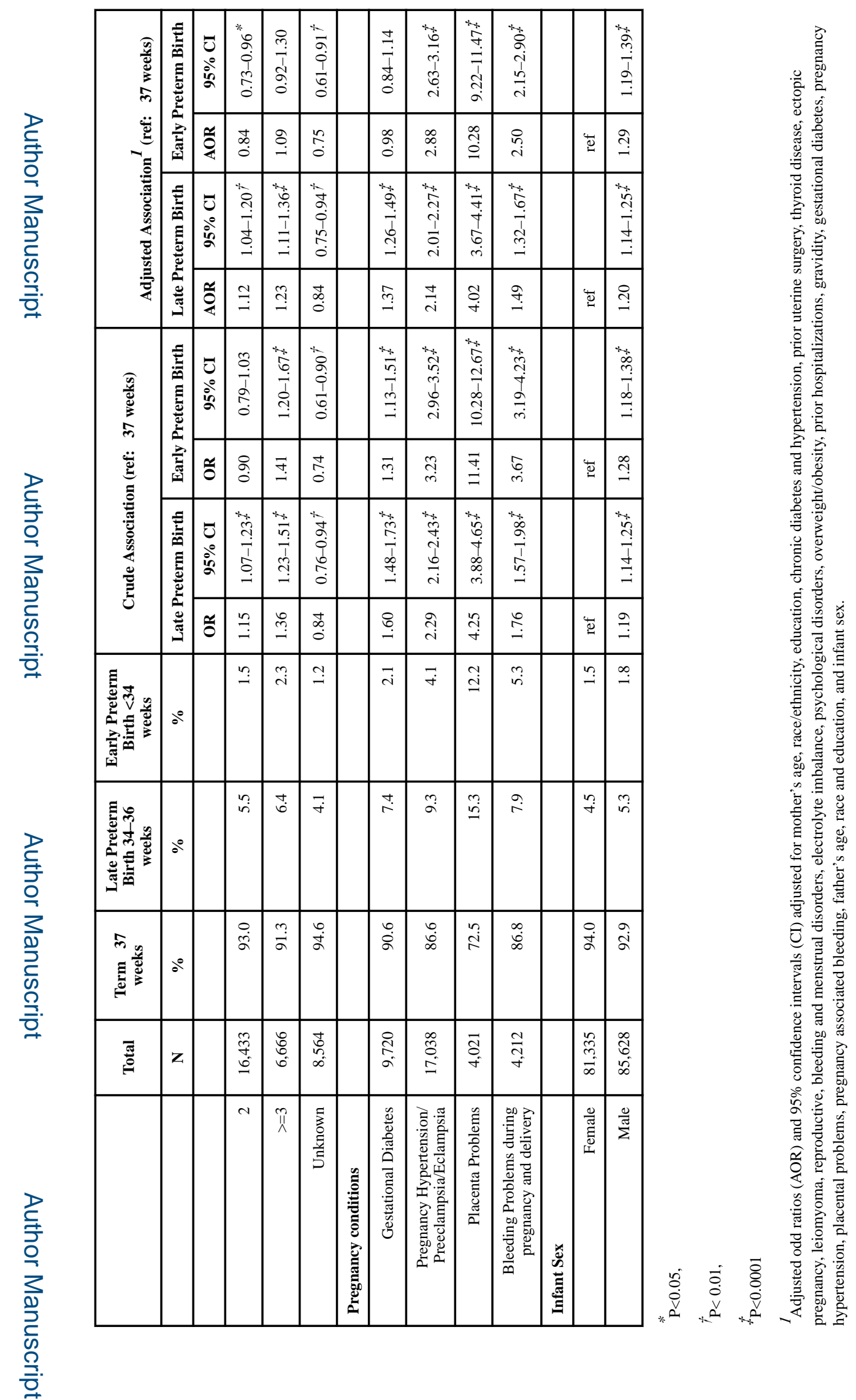

Fertil Steril. Author manuscript; available in PMC 2021 October 01. 

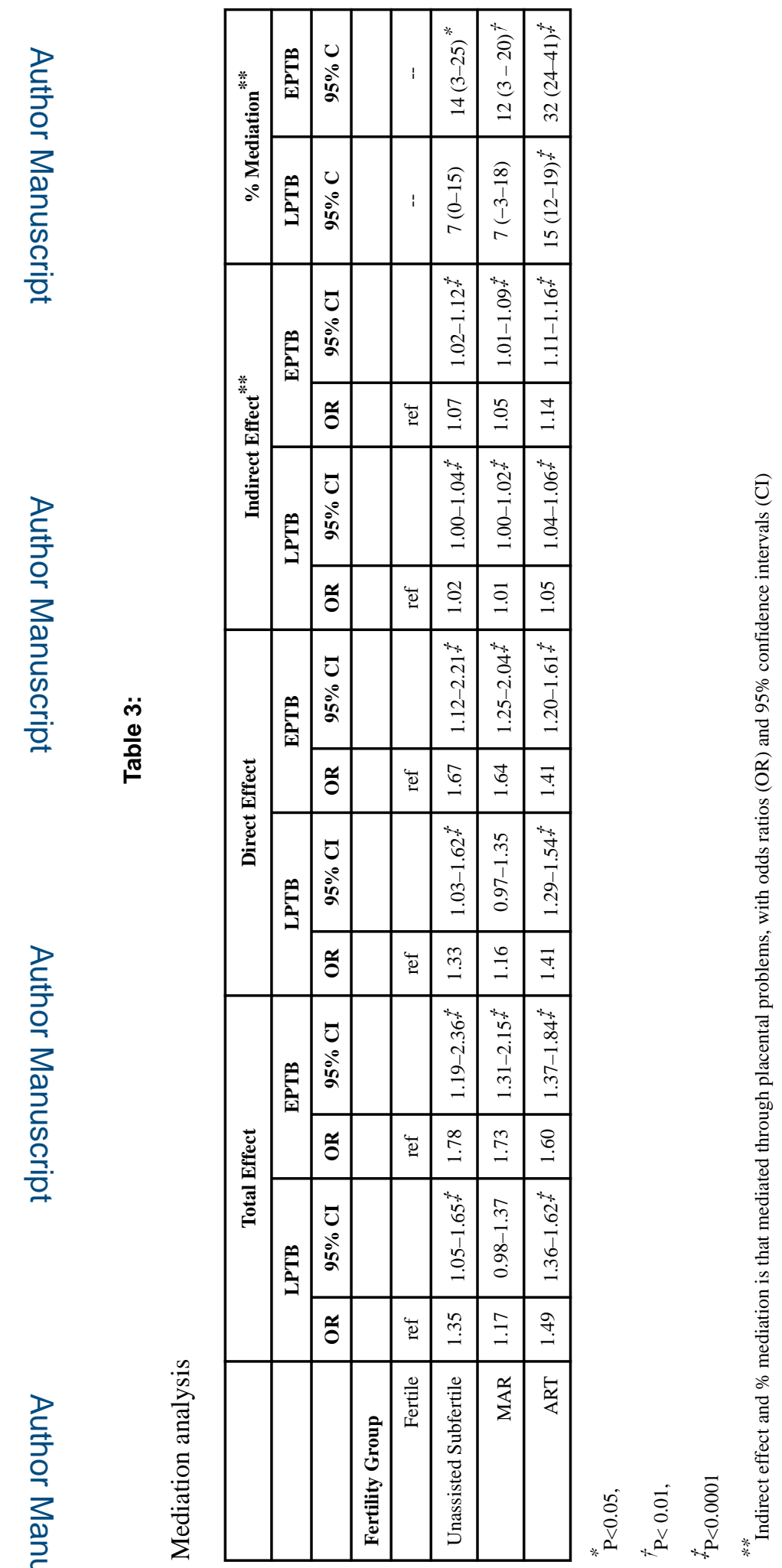

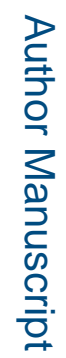

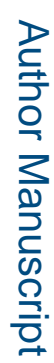

$2+x^{2} x^{2}$

Fertil Steril. Author manuscript; available in PMC 2021 October 01. 